\title{
Identification and validation of a Hedgehog pathway-based 3-gene prognostic signature for gastric cancers
}

\author{
JIANBING WU ${ }^{1}$, XI WANG ${ }^{2}$ and WEI LU ${ }^{3}$ \\ ${ }^{1}$ The Criminal Science and Technology Department, Zhejiang Police College, Hangzhou, Zhejiang 310053; \\ ${ }^{2}$ Zhejiang Key Laboratory of Gastro-Intestinal Pathophysiology and ${ }^{3}$ Medical Laboratory Center, \\ Zhejiang Hospital of Traditional Chinese Medicine, Zhejiang Chinese Medical University, \\ Hangzhou, Zhejiang 310006, P.R. China
}

Received December 8, 2017; Accepted May 31, 2018

DOI: $10.3892 / \mathrm{ol} .2018 .8945$

\begin{abstract}
Developing prognostic factors for patients with gastric cancer (GC) is crucial for the accurate identification of subgroups with distinct clinical outcomes and the development of effective treatment strategies. The aim of this study was to determine novel gene expression signatures from the hedgehog $(\mathrm{Hh})$ signaling pathway as predictors of risk with biological significance. The Cancer Genome Atlas (TCGA) GC (STAD) cohort was used as the training dataset to select for significant prognostic Hh genes. Three Hh genes, indian hedgehog $(\mathrm{IHH})$, patched 1 (PTCH1) and smoothened frizzled class receptor (SMO), were identified to be significant prognostic factors. On this basis, a 3-Hh-gene set was constructed and the high-risk patients of the training cohort were distinguished against low-risk cases [hazard ratio $(\mathrm{HR})=1.73$, $95 \%$ confidence interval $(\mathrm{CI})=1.26-2.39, \mathrm{P}=0.00069]$. Then the gene signature was externally validated in a combined dataset from Gene Expression Omnibus ( $n=631)$, and experimentally confirmed in an independent cohort of 126 clinical GC samples by immunohistochemistry (IHC). Validation in the combined GEO dataset yielded consistent results $(\mathrm{HR}=1.45$, 95\% $\mathrm{CI}=1.17-1.81, \mathrm{P}=0.00068$ ), and remained significant for stages I-III, HER2-positive and surgery alone subgroups. Subsequently, we further demonstrated that this mRNA-based gene set could be successfully transferred to an IHC-based signature in our local cohort $(\mathrm{HR}=2.04,95 \% \mathrm{CI}=1.09-3.82$, $\mathrm{P}=0.02)$. In addition, this signature served as an independent prognostic indicator for overall survival in the multivariate Cox analysis $(\mathrm{HR}=2.133,95 \% \mathrm{CI}=1.110-4.099, \mathrm{P}=0.02)$. In conclusion, we successfully generated a stable III-Hh-gene
\end{abstract}

Correspondence to: Dr Wei Lu, Medical Laboratory Center, Zhejiang Hospital of Traditional Chinese Medicine, Zhejiang Chinese Medical University, 54 Youdian Road, Shangcheng, Hangzhou, Zhejiang 310006, P.R. China

E-mail: weiluzcmu@hotmail.com

Key words: gastric carcinomas, hedgehog pathway, prognosis, gene signature model with the ability to separate patients into prognostic subgroups, which may have notable biological importance and be easily utilized clinically.

\section{Introduction}

Gastric cancer (GC), as one of the leading causes of morbidity and mortality worldwide, demonstrates a relative poor prognosis to other cancers with an overall 5-year survival rate below 30\% (1). The TNM (tumor/node/metastasis) staging system is routinely used in the classification of GC. However, even GC patients with the same stages and treatments tend to demonstrate variations in clinical outcomes. Therefore, the efficacy of TNM staging may be unable to distinguish higher and lower risk patients, and new markers accompanying this system are urgently needed to define more robust prognostic subtypes of GC (2).

With the development of high-throughput technologies, including genomics, transcriptomics and proteomics, an increasing number of prognostic molecules have been identified, but most of them cannot be clinically applied due to low effectiveness and poor reproducibility when validated in larger cohorts (3). It has been proposed that the construction of gene signature with multiple biomarkers can greatly enhance the predictive and prognostic ability over single biomarkers; a panel of gene signatures has been introduced for clinical application. Currently, several potential prognostic gene signatures have been developed for the prognosis of GC (4-8). However, these signatures always involve scores of genes, which are not clinically applicable. Furthermore, although these prognostic signatures can define high-risk GC subtypes, most of them do not contribute to the development of personalized treatment for these patients.

The hedgehog (Hh) signaling pathway acts as a driving factor in the progression of cancer and may be considered as a potential therapeutic target; considerable efforts have been performed to develop its inhibitors to treat multiple cancers, such as GC (9). Several previous studies have evaluated the prognostic significance of the Hh signaling pathway members in small scale clinical samples of GC, the prognostic power of the combination of $\mathrm{Hh}$-associated genes at a larger scale has never been reported (10-12). Therefore, we hypothesized 
that the Hh signaling pathway-based gene signature may have greater prognostic power than a single $\mathrm{Hh}$-associated gene, and may also provide more biological and clinical significance.

In this study, using The Cancer Genome Atlas (TCGA) gene expression profiling, we developed a 3 -Hh-gene signature that may classify patients with GC into prognostic subgroups with differing clinical outcomes. We further validated the potential of this signature in silico using a large cohort of combined GC transcriptomic data, and experimentally investigated these findings in clinical GC samples.

\section{Materials and methods}

Training and validation data sets. Gene expression profiling data of TCGA GC (STAD) in RSEM format (RNA-SeqV2) were downloaded from cBioPortal (http://www.cbioportal. org/) (13). TCGA STAD comprising 386 cases of GC samples with overall survival data served as the training data set. The REEM values of gene expression levels were log-transformed before further analysis.

Combined gene expression microarray datasets (GSE14210, GSE15459, GSE22377, GSE29272, GSE51105 and GSE62254) from KM Plotter (http://kmplot.com/analysis) was used for in silico validation (14). This combined data set comprised 631 GC samples with survival data was analyzed on Affymetrix Human Genome U133/U133A 2.0/HG-U133_Plus_2 platforms.

One hundred and twenty-six cases of pathologically proven primary GC specimens at stage I-III were included for experimental confirmation in our established gene classification. All the samples were obtained with from patients with GC who received standard curative surgery at Zhejiang Hospital of Traditional Chinese Medicine from January 2004 and December 2011 with informed consent. All the patients received postoperative chemotherapy (FOLFOX regimen), and no adjacent treatment was administered before surgery. The study was approved by the institutional review boards of Zhejiang Hospital of Traditional Chinese Medicine and Zhejiang Police College.

Development of prognostic Hh signature in the training data set. Nine canonical $\mathrm{Hh}$ signaling pathway-associated genes, including three secreted ligands [ $\mathrm{SHH}$, indian hedgehog $(\mathrm{IHH})$ and $\mathrm{DHH}]$, three transmembrane receptors or co-receptors [patched $(\mathrm{PTCH}) 1, \mathrm{PTCH} 2$ and smoothened frizzled class receptor (SMO)], and three transcription factors (GLI1, GLI2 and GLI3) were selected for analysis. The Cutoff Finder online tool (http://molpath.charite.de/cutoff/) was used to determine the optimized threshold of gene expression to obtain most the significant prognostic power as described before (15). According the established cutoff value, patients were divided into high- and low-risk subgroups, and were subject to univariate Cox proportional hazards analysis; The Cox regression coefficients for each gene was calculated.

The expression value $(\mathrm{G})$ of significant $\mathrm{Hh}$-associated genes identified in univariate Cox proportional hazards analysis and their corresponding Cox regression coefficient were utilized to reconstruct the prognostic gene signature, as described in the following equation: Risk score $=\sum$ Cox coefficient of gene $\mathrm{Gi} x$ expression value of gene $\mathrm{Gi}$. The patients in the training data set were divided into two groups (high- and low-risk) according to the cutoff value for risk using Cutoff Finder. Survival times were compared between the two groups using the Kaplan-Meier analysis and log-rank test.

Assessment of the prognostic Hh gene signature in the combined GEO validation data set. The potential of the Hh-gene signature for predicting the outcome of patients with $\mathrm{GC}$ in the training data set was externally invetsigated in the combined six-microarray cohort derived from the GEO database. The workflow analysis was similar to the training data set. Risk scores were determined in the validation samples based on the above equation. The coefficient values derived from the training data set were directly applied for validation in the cohort. Two subgroups were constituted based on the median risk score, and their survival times were compared with the Kaplan-Meier analysis. The analysis was performed using the KM Plotter online tool (http://kmplot.com/analysis).

\section{Experimental validation of the prognostic significance} in our GC cohort by immunohistochemistry (IHC). Immunohistochemical staining for IHH, PTCH1 and SMO on GC tumors were performed by the avidin-biotin complex method. Briefly, $4 \mu \mathrm{m}$ of sections of paraffin-embedded tissue blocks were deparafnizated and rehydrated. After blocking of endogenous peroxidases activity and heat-induced antigen-retrieval procedures, the sections were incubated with rabbit anti-human antibodies against IHH (1:150 dilution), PTCH1 (1:100 dilution) and SMO (1:100 dilution; all Abcam, Cambridge, UK) overnight at $4^{\circ} \mathrm{C}$. Then, a HRP-conjugated secondary antibody substrate (Dako, Copenhagen, Denmark) was added, and 3,3'-diaminobenzidine (DAB) was used as the chromogen.

The widely accepted German scoring system was used to evaluate the IHC staining semi-quantitatively as previously described (16). Each tumor was assigned an IHC score (from 0 to 300 ), which was determined by multiplying the intensity score by the score for the expression extent of stained cells.

Statistical analysis. As for web-based bioinformatic tools, statistical analysis was automatically obtained and conducted online. The association between clinical variables and gene expression levels generated from IHC-based validation were evaluated using the Chi-square test. Cumulative survival curves were computed by using the Kaplan-Meier method, and evaluated between groups using the log-rank test. Multivariate survival analysis was performed using Cox's regression model. All reported P-values were two-sided, and $\mathrm{P}<0.05$ was considered to indicate a statistically significant difference. All the statistical analyses were performed using SPSS 22.0 software (IBM SPSS Statistics, Armonk, NY, USA).

\section{Results}

Detection of the Hh signaling pathway-based genes is associated with overall survival and development of prognostic gene signature in the training data set. We first evaluated the prognostic values of 9 canonical Hh signaling pathway-associated genes for patients with GC in TCGA STAD data set. Three members IHH, PTCH1 and SMO were identified to have significant prognostic value at cutoff values 

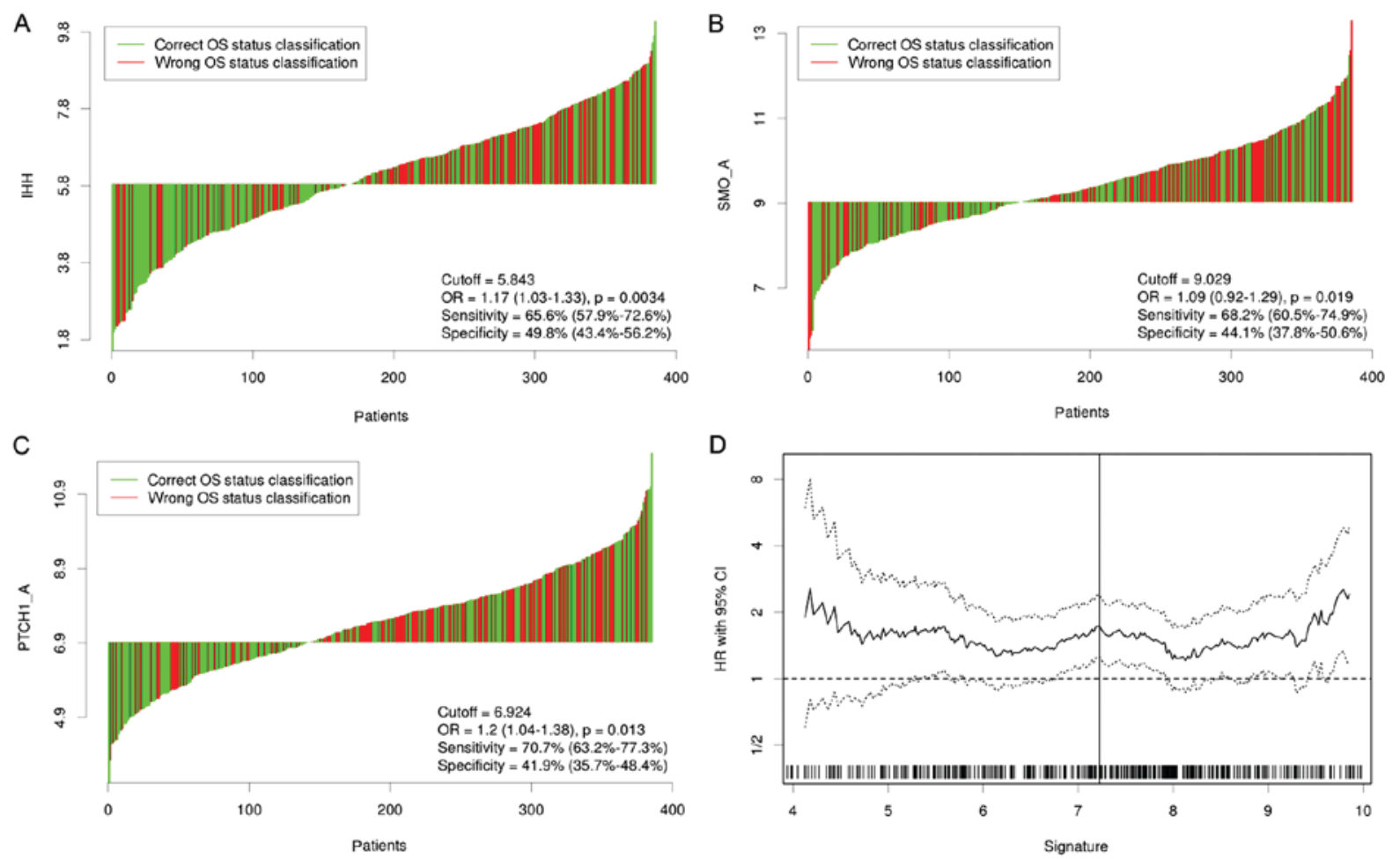

Figure 1. Optimal dichotomization of Hh genes and 3-gene signature in TCGA STAD data set. Comparison of survival status and gene expression distribution according to cutoff values of individual genes (A, IHH; B, SMO; C, PCTH1). (D) The HR including 95\% CI was plotted for each cutoff. TCGA, The Cancer Genome Atlas; IHH, indian hedgehog; PTCH1, patched 1; SMO, smoothened frizzled class receptor; HR, hazard ratio.
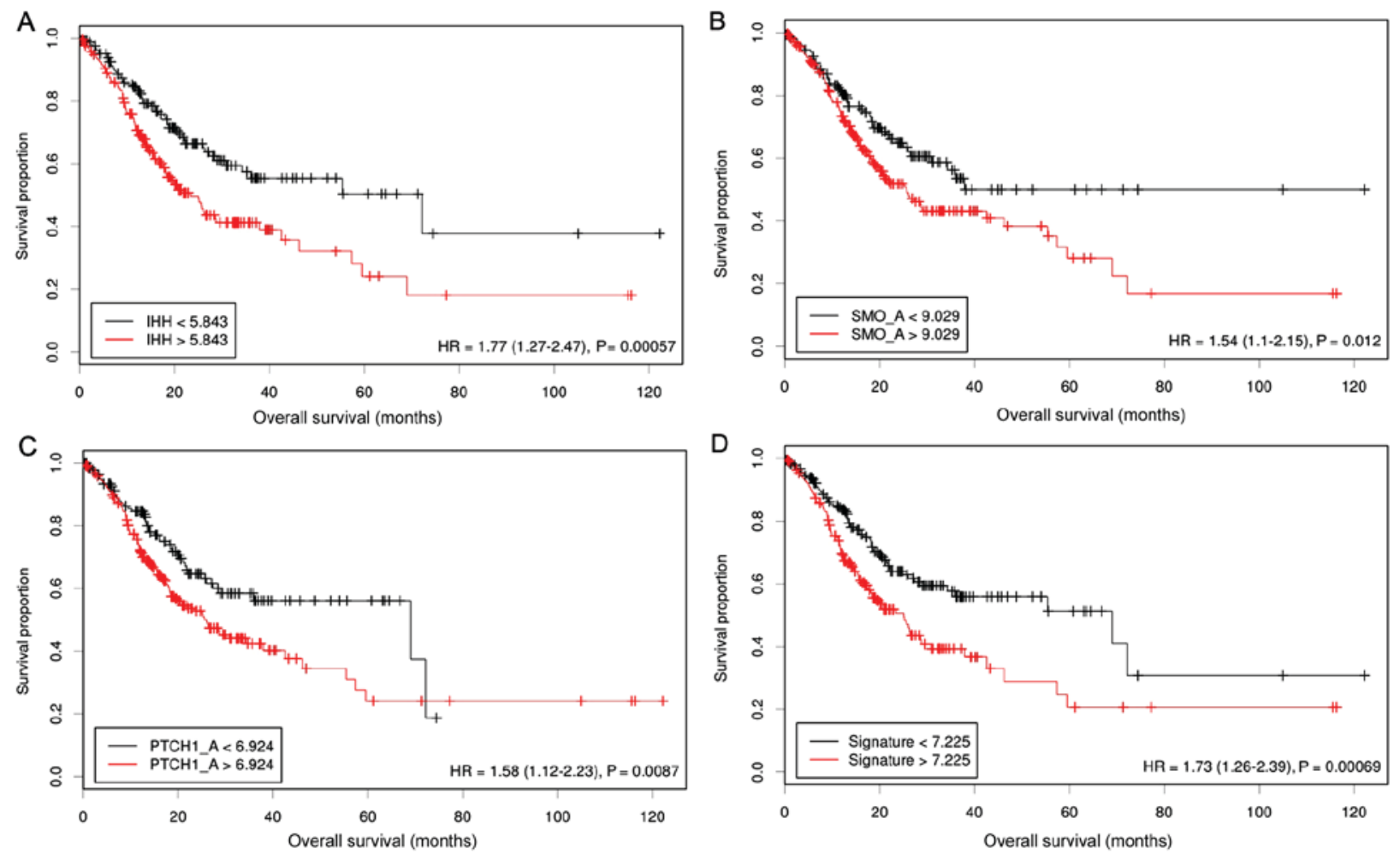

Figure 2. Kaplan-Meier analysis of the cumulative survival rate on patients dichotomised into high-risk subgroup and low-risk subgroup at the cut-off points. (A) IHH; (B) SMO; (C) PTCH1 and (D) gene signature at using the optimal cutoff. TCGA, The Cancer Genome Atlas; IHH, indian hedgehog; PTCH1, patched 1; SMO, smoothened frizzled class receptor; HR, hazard ratio.

of 5.843, 6.924 and 9,029 (Fig. 1); high expression of IHH, PTCH1 and SMO was significantly associated with mortality and shorter overall survival (Fig. 2). Subsequently, a univariate Cox regression analysis was carried out to calculate the 
coefficient for each of the three Hh-associated biomarkers. The prognostic risk for each case was then scored by summing the coefficient-weighted expression of the IHH-PTCH1-SMO signature as follows: 3-gene signature score $=(0.553 x I H H$ value $)+(0.457 x$ PTCH1 value $)+(0.411 x$ SMO value $) . \mathrm{We}$ found that this signature had the best prognostic ability at a cutoff value of 7.225; patients with higher risk scores $(n=201)$ had a significantly shorter survival than those in the lower risk group $(\mathrm{n}=186)$ [Hazard Ratio $(\mathrm{HR})=1.73,95 \%$ confidence interval $(\mathrm{CI})=1.26-2.39$, log-rank test, $\mathrm{P}=0.00069$ ] (Figs. 1 and 2). The expression levels of the 9 Hh-associated genes in the high- and low-risk subgroups were demonstrated in Fig. 3.

Prediction performance of the 3-Hh-gene signature in the combined GEO dataset. To reliably estimate the prediction potential, we extracted the expression data of these 3 Hh-associated genes from another large cohort of the GEO database. When the same coefficient in the training data set was used, patients with GC of the validation cohort were dichotomized according to the risk scores; overall survival rates were significantly elevated in the subgroup with high risk scores (HR=1.45, 95\% CI=1.17-1.81, $\mathrm{P}=0.00068)$ (Fig. 4).

To test whether the 3-gene signature is independent of the current staging systems and other prognostic predictors, the validation cohort were further stratified according to stages, HER2 status and treatment therapies. We found that the risk score successfully separated patients into prognostic subgroups in all the stages except stage IV (Fig. 4). In addition, we also observed that this gene signature may also be effectively used to identify high-risk individuals in the HER2-negative subgroup $(\mathrm{HR}=1.65,95 \% \mathrm{CI}=1.26-2.16, \mathrm{P}=0.00024)$ and patients with $\mathrm{GC}$ receiving surgery alone $(\mathrm{HR}=1.49,95 \% \mathrm{CI}=1.11-1.99$, $\mathrm{P}=0.0075$ ). These findings indicate that the 3 -Hh-gene signature may provide more prognostic information to the current classification systems.

Experimental validation of the 3-Hh gene signature in discriminating high-risk GC patients by IHC. Next, we evaluated whether this mRNA-based gene signature retained its effectiveness in an independent cohort by the IHC-detected strategy. Representative staining images of IHH, PTCH1 and SMO were demonstrated in Fig. 5A. Each case was scored; univariate Cox regression analysis indicated that high expression levels of these markers were associated with decreased overall survival, but only reaching marginal significance. We then derived the same formula in training dataset to calculate a risk score for each case based on IHC scores. Using the formula, our cohort was divided into high$(n=39)$ and low-risk $(n=76)$ subgroups with an optimal cutoff of 2.04 using the Cutoff Finder tool (Fig. 5B). Distribution of the demographic and clinicopathological characteristics did not significantly vary between the high- and the low-risk subgroups (Table I). Furthermore, we found that patients with high-risk scores exhibited shorter survival compared with in those with low-risk scores (HR=2.04, 95\% CI=1.09-3.82-, $\mathrm{P}=0.02$ ) (Fig. 5C). In the multivariate Cox regression analyses, age, tumor size and high-risk scores of gene signature were independently associated with shorter overall survival $(\mathrm{HR}=2.133,95 \% \mathrm{CI}=1.110-4.099, \mathrm{P}=0.02)$ (Table II).
Table I. Clinical characteristics of GC patients dichotomised by IHC-detected gene signature in experimental validation cohort.

\section{3-Hh-gene signature}

Characteristics High risk $(n=39)$ Low risk $(n=76)$ P-value

\begin{tabular}{lccc}
\hline Age (years) & $66.82 \pm 11.016$ & $63.05 \pm 10.611$ & 0.805 \\
Sex & & & \\
Male & 23 & 52 & 0.314 \\
Female & 16 & 24 &
\end{tabular}

Differentiation

grade

Well or

moderately

Poorly

Tumor size

$\begin{array}{llll}\text { T1-2 } & 14 & 18 & 0.483 \\ \text { T3-4 } & 25 & 58 & \end{array}$

Lymph node

$\begin{array}{llll}\text { Negative } & 17 & 28 & 0.166 \\ \text { Positive } & 22 & 48 & \end{array}$

TNM Stage

$\begin{array}{llll}\text { I } & 11 & 11 & \\ \text { II } & 13 & 26 & 0.178 \\ \text { III } & 15 & 39 & \end{array}$

TNM, tumor node metastasis; GC, gastric cancer; IHC, immunohistochemistry.

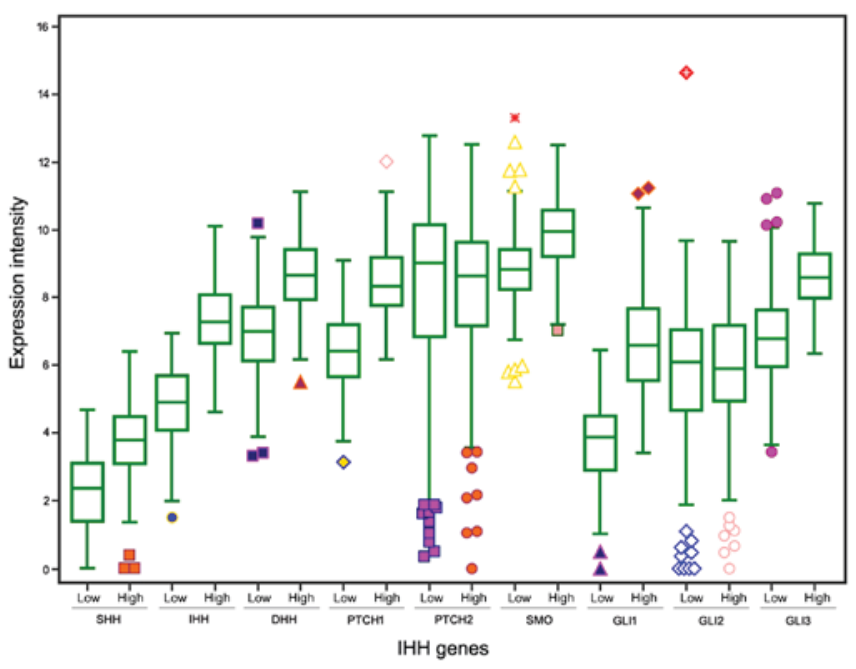

Figure 3. The expression levels of the 9 Hh-associated genes in the high- and low- risk subgroups. Three signature genes IHH, PTCH1 and SMO demonstrated significant difference in expression levels between subgroups. IHH, indian hedgehog; PTCH1, patched 1; SMO, smoothened frizzled class receptor.

\section{Discussion}

Aberrant activation of the Hh signaling pathway has been associated with a variety of human cancers, including GC (17-19). As potential targets in the treatment of GC, the 
A

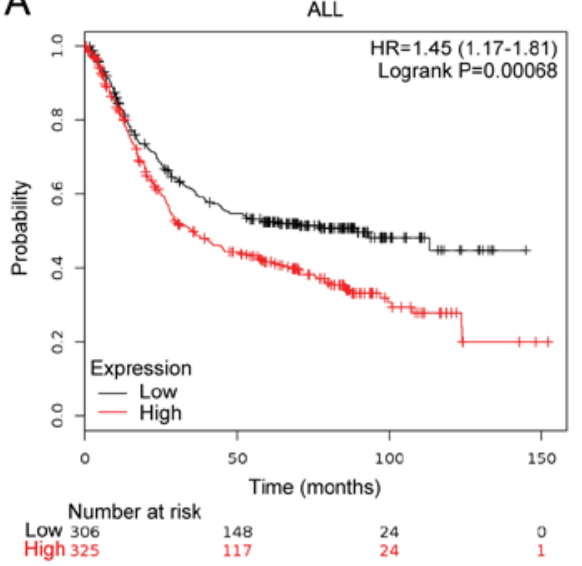

D
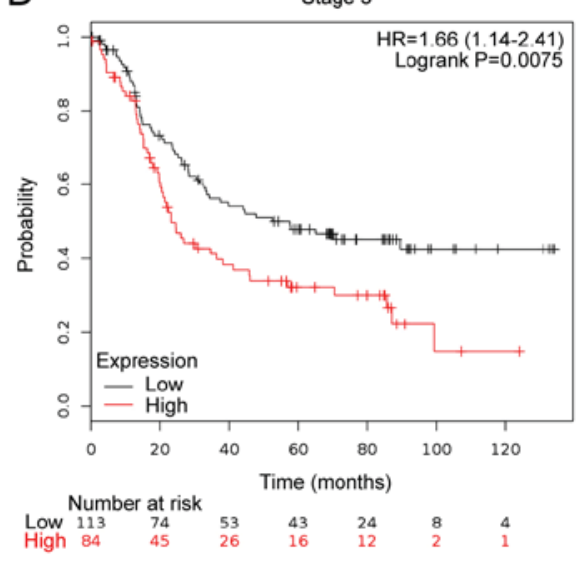

G HER2-positive

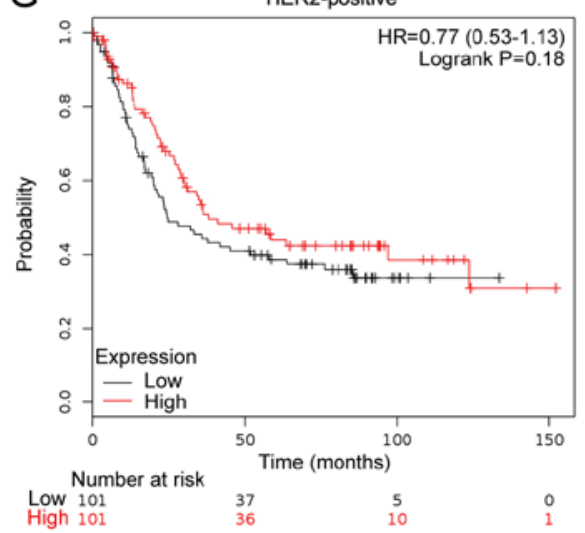

B

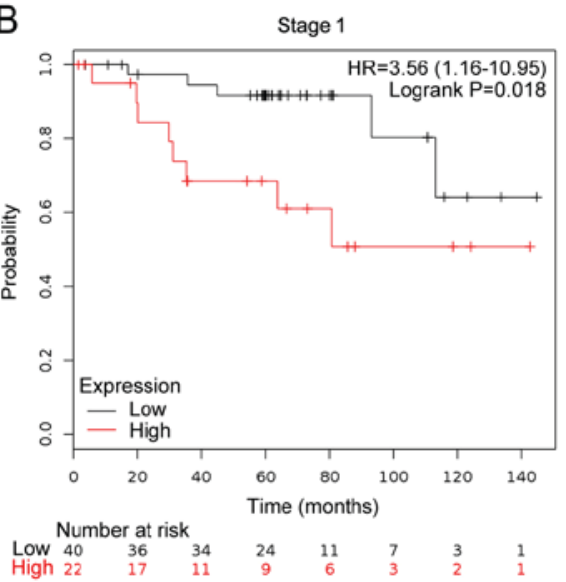

E

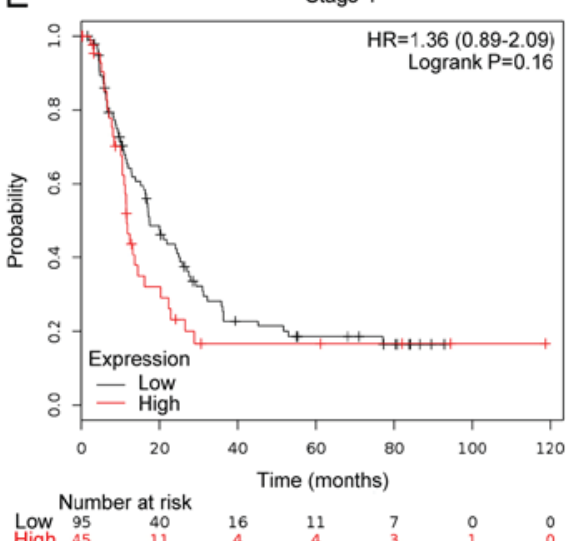

$\mathrm{H}$

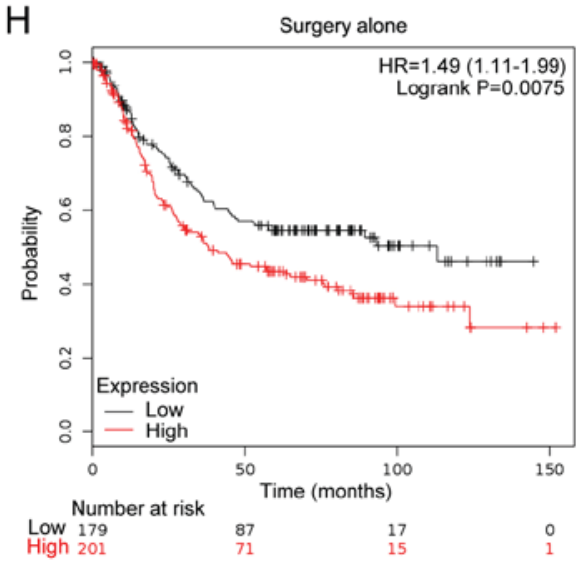

C

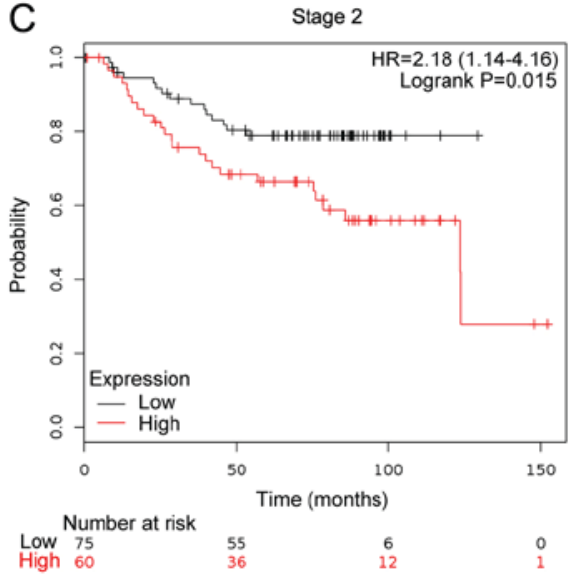

$\mathrm{F}$

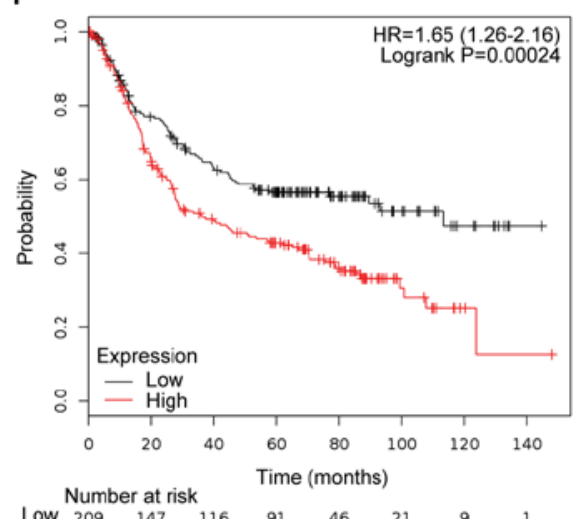

Low 209 Number at risk

I

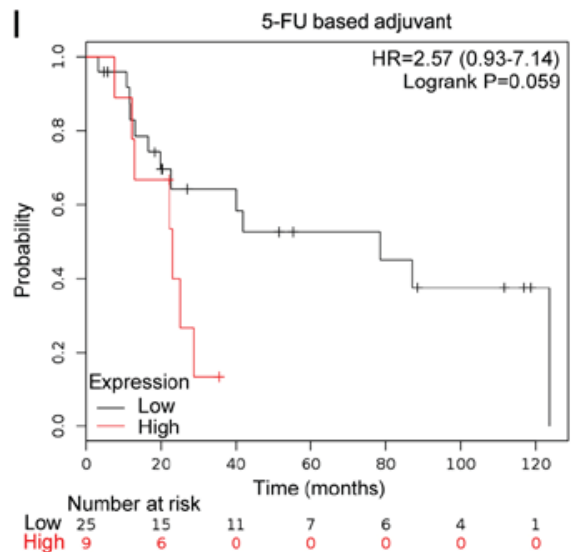

Figure 4. The association between the risk subgroups defined by 3-gene signature in the combined GEO cohort. This gene signature demonstrated the ability to separate patients into prognostic subgroups (A), and retain significant in subgroups of stage I-III (B-D), HER2-negative (F), and surgery alone (H), but not in stage IV (E), HER2-positive (G), and 5-FU adjuvant patients (I). HR, hazard ratio.

prognostic impact of the Hh signaling pathway genes in GC have been widely explored previously. However, a potent, reproducible $\mathrm{Hh}$-associated prognostic factor for clinical use is yet to be confirmed. This is may be due to the fact that most the previous prognostic studies of Hh-associated factors in GC are limited to small cohorts and focus on signle not combined Hh biomarker in combination.

To overcome these limitations, in this study, we used two large gene expression cohorts (both comprising more than 300 samples with survival data) to identify and validate the most potent Hh-associated pathway factors for the prognoses of GC. Among current canonical Hh-associated genes, we identified IHH, PTCH1 and SMO as the most powerful prognostic factors for GC. Furthermore, we developed a signature with these three Hh-associated genes, which demonstrated a stable and reliable potential to identify high-risk GC patients with poor clinical outcome. These reproducible effects were confirmed in a variety independent cohorts and different gene expression platforms (RNA-seq, microarray, and IHC). Furthermore, we demonstrated that this signature was independent of known clinical prognostic factors, and in particular, its robustness was effective in early and advanced stages; HER2-negative or surgery alone patients exhibited the most heterogeneous clinical outcomes. HER2-positive 
Table II. Cox regression analysis of IHC-based gene signature score and clinicopathological covariates with overall survival.

\begin{tabular}{|c|c|c|c|c|}
\hline \multirow[b]{2}{*}{ Characteristics } & \multicolumn{2}{|c|}{ Univariate analysis } & \multicolumn{2}{|c|}{ Multivariate analysis } \\
\hline & HR $(95 \% \mathrm{CI})$ & P-value & HR $(95 \% \mathrm{CI})$ & P-value \\
\hline Age & $1.044(1.011-1.078)$ & 0.009 & $1.041(1.110-1.076)$ & 0.016 \\
\hline Sex & $0.883(0.458-1.701)$ & 0.710 & $0.742(0.382-1.443)$ & 0.379 \\
\hline Grade & $1.550(0.836-2.874)$ & 0.164 & $1.659(0.883-3.120)$ & 0.116 \\
\hline Tumor size & $2.471(1.036-5.890)$ & 0.041 & $3.182(1.268-7.986)$ & 0.014 \\
\hline Lymph node & $1.081(0.567-2.061)$ & 0.812 & $0.755(0.378-1.509)$ & 0.427 \\
\hline 3-Hh-gene signature & $1.957(1.045-3.661)$ & 0.036 & $2.133(1.110-4.099)$ & 0.023 \\
\hline
\end{tabular}

HR, hazard ratio; CI, confidence interval; IHC, immunohistochemistry; Hh, hedgehog.

A

High risk
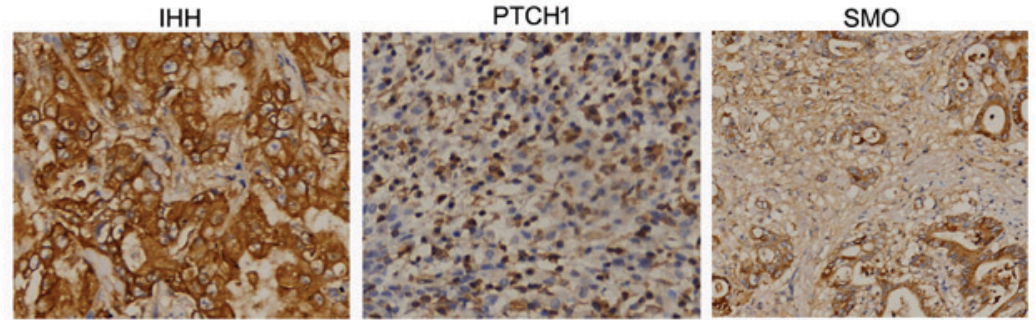

Low risk
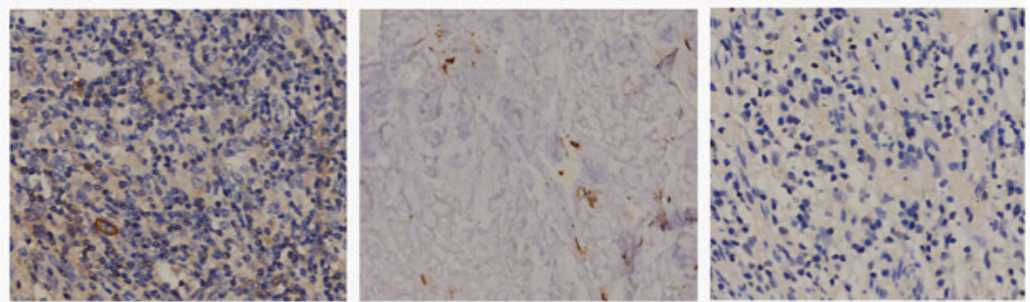

B

Cutoff $=177.5,39(33.9 \%)$ score+, $76(66.1 \%)$ score.

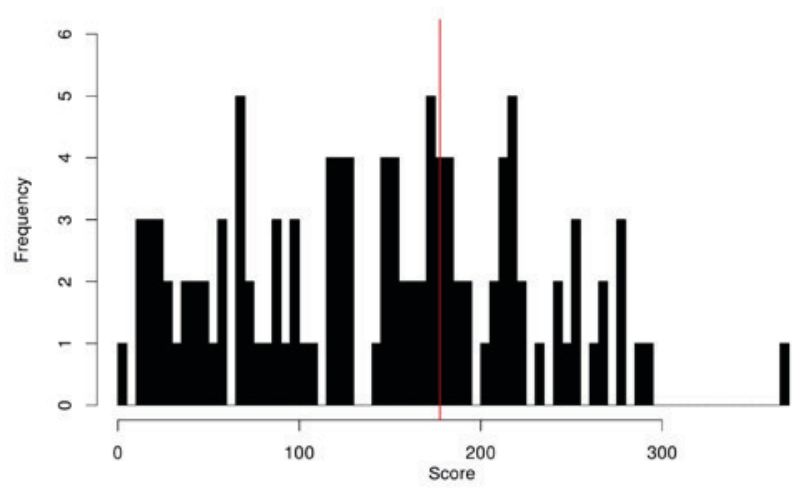

C

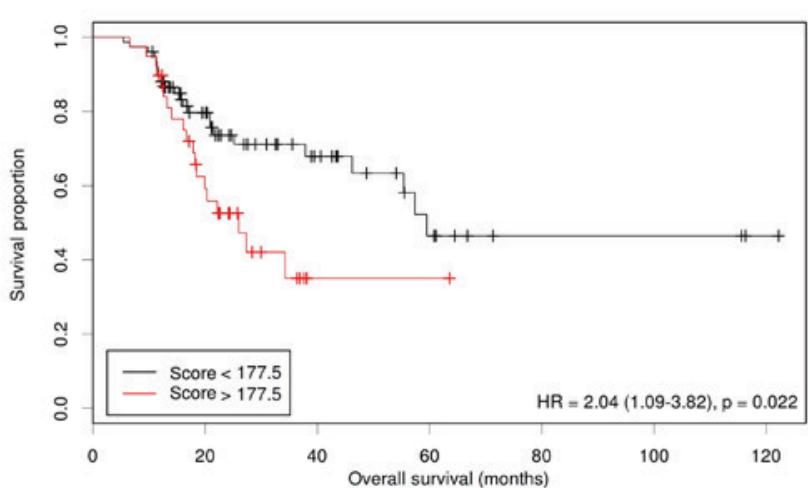

Figure 5. Validation of 3-gene-signature in IHC cohort. (A) Representative images of immunostaining at high- and low- risk subgroups (magnification, x100). (B) IHC score distribution and cutoff determined by Cutoff Finder software. (C) Kaplan-Meier analysis of the cumulative survival rate in terms of risk classification at a cutoff of 177.5. IHC, immunohistochemistry; PTCH1, patched 1; SMO, smoothened frizzled class receptor. 
GCs represent a subgroup with poor prognosis that can be treated using anti-HER2 drugs, such as trastuzumab (20). Our signature further identified a poor-prognostic subgroup from the HER2-negative GCs, but did not benefit from treatment with anti-HER 2 drugs; therefore, alternative treatments may be considered for these patients. Therefore, our signature may provide more details for the current risk classification system.

Compared with previous established prognostic gene signatures, our signature only has 3 genes, which is simple and practical for further clinical use. Secondly, although our 3-Hh-gene signature is derived from RNA-seq and microarray-based platforms, in this study, we also identified a high-risk GC subgroup with poor prognosis by using simple IHC technology. Therefore, this signature may have notable biological importance. PTCH1 has been reported to be closely associated with the chemoresistance of GC cell lines (21). Recently, Ma et al (22) linked high SMO expression levels with paclitaxel-resistant GC clinical samples; IPI-926, an inhibitor of SMO, may sensitize paclitaxel-resistant tumors. SMO has been confirmed to be a target with the highest therapeutic potential; a panel of SMO inhibitors have been investigated for the treatment of cancer $(23,24)$. Therefore, it may be reasonable to speculate that high-risk patients defined by our 3 -Hh gene signature may benefit most from SMO inhibitors.

The limitation of the present study is that this signature was investigated in a retrospective manner, therefore, future study may require the integration of this model with prospective randomized trials to further validate its clinical relevance.

In summary, we identified a stable Hh signaling pathway-based 3 gene set that may provide additional prognostic information to improve clinical classification, and transfer to IHC-based assay. We propose that it may be developed into a robust, practical and inexpensive molecular diagnostic tool for clinical use in the near future.

\section{Acknowledgements}

Not applicable.

\section{Funding}

The present study was supported by Key Research Program of Science and Technology Planning Project of Zhejiang Province (grant no. 2017C3026) and Zhejiang Provincial Natural Science Foundation of China (grant no. LQ14H160014).

\section{Availability of data and materials}

All data generated or analyzed during this study are included in this published article.

\section{Authors' contributions}

WL and JW designed the project and were responsible for the data analysis. WL and XW performed the IHC experiments. JW and WL wrote the manuscript. All authors read and approved the final manuscript.

\section{Ethics approval and consent to participate}

The study was approved by the institutional review boards of Zhejiang Hospital of Traditional Chinese Medicine and Zhejiang Police College. Written informed consent was obtained from all patients.

\section{Consent for publication}

Not applicable.

\section{Competing interests}

The authors declare no competing interests.

\section{References}

1. Miller KD, Siegel RL, Lin CC, Mariotto AB, Kramer JL, Rowland JH,Stein KD, Alteri R and Jemal A: Cancer treatment and survivorship statistics, 2016. CA Cancer J Clin 66: 271-289, 2016.

2. Figueiredo C, Camargo MC, Leite M, Fuentes-Pananá EM, Rabkin CS and Machado JC: Pathogenesis of gastric cancer: Genetics and molecular classification. Curr Top Microbiol Immunol 400: 277-304, 2017.

3. Ghosh D and Poisson LM: 'Omics' data and levels of evidence for biomarker discovery. Genomics 93: 13-16, 2009.

4. Cho JY, Lim JY, Cheong JH, Park YY, Yoon SL, Kim SM, Kim SB, Kim H, Hong SW, Park YN, et al: Gene expression signature-based prognostic risk score in gastric cancer. Clin Cancer Res 17: 1850-1857, 2011.

5. Wang P, Wang Y, Hang B, Zou X and Mao JH: A novel gene expression-based prognostic scoring system to predict survival in gastric cancer. Oncotarget 7: 55343-55351, 2016.

6. Hou JY, Wang YG, Ma SJ, Yang BY and Li QP: Identification of a prognostic 5-Gene expression signature for gastric cancer. J Cancer Res Clin Oncol 143: 619-629, 2017.

7. Lee J, Cristescu R, Kim KM, Kim K, Kim ST, Park SH and Kang WK: Development of mesenchymal subtype gene signature for clinical application in gastric cancer. Oncotarget 8: 66305-66315, 2017.

8. Wang X, Liu Y, Niu Z, Fu R, Jia Y, Zhang L, Shao D, Du H, Hu Y, Xing $\mathrm{X}$, et al: Prognostic value of a 25 -gene assay in patients with gastric cancer after curative resection. Sci Rep 7: 7515, 2017.

9. Wu F, Zhang Y, Sun B, McMahon AP and Wang Y: Hedgehog signaling: From basic biology to cancer therapy. Cell Chem Biol 24: 252-280, 2017.

10. Abdel-Rahman O: Hedgehog pathway aberrations and gastric cancer; evaluation of prognostic impact and exploration of therapeutic potentials. Tumour Biol 36: 1367-1374, 2015.

11. Lee SJ, Do IG, Lee J, Kim KM, Jang J, Sohn I and Kang WK: Gastric cancer (GC) patients with hedgehog pathway activation: PTCH1 and GLI2 as independent prognostic factors. Target Oncol 8: 271-280, 2013.

12. Wang ZS, Shen Y, Li X, Zhou CZ, Wen YG, Jin YB and Li JK: Significance and prognostic value of Gli-1 and Snail/E-cadherin expression in progressive gastric cancer. Tumour Biol 35: 1357-1363, 2014.

13. Gao J, Aksoy BA, Dogrusoz U, Dresdner G, Gross B, Sumer SO, Sun Y, Jacobsen A, Sinha R, Larsson E, et al: Integrative analysis of complex cancer genomics and clinical profiles using the cBioPortal. Sci Signal 6: pl1, 2013.

14. Szász AM, Lánczky A, Nagy Á, Förster S, Hark K, Green JE, Boussioutas A, Busuttil R, Szabó A and Gyórffy B: Cross-validation of survival associated biomarkers in gastric cancer using transcriptomic data of 1,065 patients. Oncotarget 7 : 49322-49333, 2016.

15. Budczies J, Klauschen F, Sinn BV, Győrffy B, Schmitt WD Darb-Esfahani S and Denkert C: Cutoff finder: A comprehensive and straightforward Web application enabling rapid biomarker cutoff optimization. PLoS One 7: e51862, 2012.

16. Ishibashi H, Suzuki T, Suzuki S, Moriya T, Kaneko C, Takizawa T, Sunamori M, Handa M, Kondo T and Sasano H: Sex steroid hormone receptors in human thymoma. J Clin Endocrinol Metab 88: 2309-2317, 2003. 
17. Peukert S and Miller-Moslin K: Small-molecule inhibitors of the hedgehog signaling pathway as cancer therapeutics. ChemMedChem 5: 500-512, 2010.

18. Wan J, Zhou J, Zhao H, Wang M, Wei Z, Gao H, Wang Y and Cui H: Sonic hedgehog pathway contributes to gastric cancer cell growth and proliferation. Biores Open Access 3: 53-59, 2014.

19. Lee DH, Lee SY and Oh SC: Hedgehog signaling pathway as a potential target in the treatment of advanced gastric cancer. Tumour Biol 39: 1010428317692266, 2017.

20. Bang YJ, Van Cutsem E, Feyereislova A, Chung HC, Shen L, Sawaki A, Lordick F, Ohtsu A, Omuro Y, Satoh T, et al: Trastuzumab in combination with chemotherapy versus chemotherapy alone for treatment of HER2-positive advanced gastric or gastro-oesophageal junction cancer (ToGA): A phase 3, open-label, randomised controlled trial. Lancet 376: 687-697, 2010.

21. Yu B, Gu D, Zhang X, Li J, Liu B and Xie J: GLI1-mediated regulation of side population is responsible for drug resistance in gastric cancer. Oncotarget 8: 27412-27427, 2017.
22. Ma H, Tian $\mathrm{Y}$ and $\mathrm{Yu} \mathrm{X:} \mathrm{Targeting} \mathrm{smoothened} \mathrm{sensitizes}$ gastric cancer to chemotherapy in experimental models. Med Sci Monit 23: 1493-1500, 2017

23. Khoo AB, Ali FR and Lear JT: Defining locally advanced basal cell carcinoma and integrating smoothened inhibitors into clinical practice. Curr Opin Oncol 28: 180-184, 2016.

24. Doan HQ, Silapunt S and Migden MR: Sonidegib, a novel smoothened inhibitor for the treatment of advanced basal cell carcinoma. Onco Targets Ther 9: 5671-5678, 2016.

This work is licensed under a Creative Commons Attribution-NonCommercial-NoDerivatives 4.0 International (CC BY-NC-ND 4.0) License. 